\title{
HONGOS DE MICORRIZA ARBUSCULAR EN TRES AGROECOSISTEMAS DE CACAO (Theobroma cacao L.) EN LA AMAZONÍA PERUANA
}

\author{
Krystel Clarissa ROJAS MEGO ${ }^{1}$, Christyn ELIZARBE MELGAR ${ }^{1}$, Merlin Harold GÁRATE DÍAZ ${ }^{1}$, Diana \\ AYALA MONTEJO², Pedro RUIZ CUBILLAS ${ }^{3}$, Ewald SIEVERDING ${ }^{4}$ \\ 1. Programa de Investigación en Manejo Integral del Bosque y Servicios Ambientales. Instituto de Investigaciones de la \\ Amazonía Peruana. Carretera Federico Basadre. Km. 12.400-Pucallpa-Perú. e-mail: krojas@iiiap.org.pe. \\ 2. DyG Solución biorganica S.A.C., Especialista en bioremediación de suelos. e-mail:diayalamontejo@gmail.com \\ 3. Grupo PGR IMOGS Environmental S.A.C., Especialista en Micorrizas y Restauración de Suelos. e-mail: \\ pruiz@pgrimogs.com \\ 4. Institute of Plant Production and Agroecology in the Tropics and Subtropics. University of Hohenheim, Stuttgart, \\ Germany. Especialista en Identificación de Hongos de Micorriza Arbuscular. e-mail: ewald.sieverding@evonik.com
}

\section{RESUMEN}

El presente estudio tuvo como objetivo conocer la abundancia y diversidad de Hongos de Micorriza Arbuscular (HMA) asociados con cacao Theobroma cacao L. en tres diferentes agroecosistemas (AGR), ubicados en el distrito de Irazola, provincia de Padre Abad, región Ucayali, en la Amazonía peruana. Los AGR incluyeron: 1) Cacao en monocultivo (AGR-1), 2) Cacao asociado con Inga edulis - guaba (AGR-2), y 3) cacao con cobertura de kudzu (Pueraria phaseoloides) (AGR-3). Se realizaron cuatro evaluaciones del número de esporas de HMA durante el período de julio a octubre del 2013. La abundancia fue mayor en el AGR-3, con 1100 a 780 esporas por 100 gr de suelo, seguido por los AGR-2 y AGR-1; sin embargo, se encontró mayor diversidad en el AGR-2 identificándose 29 especies, seguido por los AGR-1 y AGR-3. Las especies de HMA identificadas pertenecían a los géneros Acaulospora, Ambispora, Archeospora, Cetraspora, Clareideoglomus, Diversispora, Fuscutata, Glomus, Kuklospora, Pacispora, Paraglomus y Sclerocystis. Se concluye que el número de esporas esta relacionado con la densidad de la cobertura, mientras la diversidad de especies HMA parece estar asociada con la diversidad de especies de plantas, siempre y cuando ninguna especie vegetal domine la cobertura.

PALABRAS CLAVE: Amazonía, diversidad de especies vegetales, sistemas de producción, Pueraria phaseoloides.

\section{FUNGI OF ARBUSCULAR MYCORRHIZAL IN THREE COCOA (Theobroma cacao L.) AGROECOSYSTEMS IN THE PERUVIAN AMAZON}

\begin{abstract}
The objective of the present study was to know the abundance and diversity of AMF associated with three different cacao (Theobroma cacao L.) agroecosystems (AGR), located in the district of Irazola, province of Padre Abad, Region Ucayali in the Peruvian Amazon. Cacao AGR included: 1) Cacao in monoculture associated with some other tropical trees; 2) cacao associated with Inga edulis (guaba) and many other forest species, and 3) Cacao associated with many forest plant species and with a denser legume cover of kudzu (Pueraria phaseoliodes). The number of spores of the fungi were evaluated four times during the period of july to october, 2013. Results obtained showed that spore abundance was greater in AGR-3 with 1100-780 spores / $100 \mathrm{~g}$ of soil, followed by AGR-2 and AGR-1. Fungal species diversity was greater in AGR 2, with 29 species, followed by AGR 1 and AGR-3 with de 16 and 13 species, respectively. AMF species identified belonged to the genera Acaulospora, Ambispora, Archeospora, Cetraspora, Clareideoglomus, Diversispora, Fuscutata, Glomus, Kuklospora, Pacispora, Paraglomus y Sclerocystis. It is concluded that the main factors that could influence the spore abundance cacao ecosystems is the density of ground coverage ecosystems is the density of ground coverage, here with kudzu. AMF ecosystems is the density of ground coverage, here ecosystems is the density of ground coverage, here with kudzu. AMF species diversity is determined by plant species diversity without being one plant species dominating in soil coverage. This was the case in AGR-2.
\end{abstract}

KEYWORDS: Amazon, plant diversity, production systems, Pueraria phaseoloides. 


\section{INTRODUCCIÓN}

El cultivo del cacao en la Amazonía del Perú está creciendo progresivamente debido a las condiciones agroecológicas que promueven su desarrollo, la importante demanda que tiene en el mercado y la promoción y apoyo al agricultor por parte de las instituciones del estado y otras meramente privadas. Los últimos reportes del Ministerio de Agricultura indican un crecimiento de mil hectáreas del $2010 \mathrm{al}$ 2011, además menciona que los departamentos de San Martin y Ucayali presentan los rendimientos más altos en $\mathrm{T} / \mathrm{Ha}$ correspondiente a $0,9 \mathrm{~T} / \mathrm{Ha}$.

En tal sentido, es necesario realizar esfuerzos que permitan mantener la calidad del suelo, su composición física, química y biológica, conocida como biota, la cual alberga una gran diversidad de microorganismos.

Dentro de los microorganismos de mayor uso se destacan los HMA, que son microorganismos del suelo que forman una asociación simbiótica mutualista con aproximadamente un $80 \%$ de las plantas terrestres.

La importancia de los HMA en la agricultura radica en que, por su extenso micelio extra radical, favorecen el vínculo existente entre la planta y el suelo, debido a que al darse la asociación plantahongo, se incrementa la absorción de nutrientes de poca movilidad (como el P), factor determinante que no ocurre en las plantas no micorrizadas.

En las plantas micorrizadas, el micelio externo se extiende a una mayor distancia en el suelo que los pelos radicales de las plantas no micorrizadas (Blanco y Salas, 1997). El papel de las micorrizas en la rizósfera de las plantas favorece la nutrición mineral, principalmente en cuatro aspectos: fisiología y desarrollo de la planta, crecimiento y morfología de las raíces, procesos de absorción y disponibilidad de nutrimentos (Blanco y Salas, 1997; Medina y Azcón, 2010).

Es un hecho universalmente aceptado que las micorrizas estimulan el crecimiento, desarrollo y nutrición de las plantas, especialmente en suelos de baja y moderada fertilidad (Medina y Azcón, 2010).

En los sistemas agroforestales, los HMA forman redes hifales de intercambio de nutrientes entre plantas del mismo o diferente género. Las redes ocurren por fusión de hifas (anastomosis) (Iglesias, Salas, Leblanc y Nygren, 2010). Hasta hace poco se creía que este fenómeno ocurría únicamente entre HMA de la misma especie fúngica y de la misma zona geográfica.

Sin embargo, recientemente se han presentado evidencias de anastomosis funcional entre HMA genéticamente diferentes que provenían de la misma población (Iglesias et al., 2010). De esta forma, es posible el intercambio de nutrientes entre árboles de diferente género vía conexiones hifales de HMA pertenecientes a la misma población. La probabilidad de que esto ocurra es alta, si se considera la ausencia de especificidad de los HMA hacia sus hospederos (McGonigle y Fitter, 1990).

Por ser una planta micótrofa obligada, el cacao requiere de un manejo conservacionista que promueva la simbiosis hongo-raíz.

En este sentido, se desarrolló el presente estudio, con el objetivo de determinar la abundancia y diversidad de HMA en tres agroecosistemas de cacao (Theobroma cacao Linneo), fijar su potencial y detectar prácticas y asociaciones que contribuyan al incremento de la población micorrícica.

\section{MATERIALES Y MÉTODOS}

\section{Ubicación}

El trabajo se desarrolló en tres tipos de agroecosistemas de cacao: monocultivo $\left(8^{\circ} 43^{\prime} 45.09^{\prime \prime S} 75^{\circ} 6^{\prime} 11.01 " \mathrm{O}\right)$, asociado con guaba (Inga edulis) $\left(8^{\circ} 42^{\prime} 53.98^{\prime \prime S} 75^{\circ} 5^{\prime} 9.05^{\prime \prime O}\right)$ у asociado con cobertura Kudzu (Pueraria phaseloides $)\left(8^{\circ} 47^{\prime} 14.66^{\prime \prime S} 75^{\circ} 3^{\prime} 4.88^{\prime \prime} \mathrm{O}\right)$, pertenecientes a la Asociación de Cacaoteros Tecnificados Padre Abad - ACATPA, distrito de Irazola, provincia de Padre Abad, departamento de Ucayali-Perú.

\section{Aspectos edafoclimáticos}

La temperatura promedio de la zona de estudio es de $26.7^{\circ} \mathrm{C}$; la temperatura máxima promedio es de $29.3{ }^{\circ} \mathrm{C}$ y la mínima promedio es de $24{ }^{\circ} \mathrm{C}$. La humedad relativa promedio es de $78,9 \%$.

La precipitación anual promedio es de $3600 \mathrm{~mm}$ con una estación muy lluviosa (noviembre - marzo) y otra de menor precipitación (abril - octubre) (Flores, 2010).

En la tabla 1 se muestran las variables meteorológicas de temperatura y precipitación durante el período de estudio.

Los suelos son de origen sedimentario, de textura arcillosa a arcillo-arenosa, drenaje pobre, fácilmente compactables y $\mathrm{pH}$ promedio de 5,1 .

Según la clasificación FAO, en la zona de estudio existen los siguientes tipos de suelos: Gleysol, Acrisol y Cambisol (Inceptisol) (Flores, 2010).

La tabla 2 muestra las propiedades físicoquímicas de las parcelas estudiadas. 


\section{Descripción de agroecosistemas}

Agroecosistema 1 (denominado "Monocultivo, AGR-1")

Plantación de siete años de edad bajo distanciamiento de $3 \times 3 \mathrm{~m}$, ubicada en condiciones de topografía accidentada, en la que se realiza deshierbo mecánico cada cuatro meses y poda sanitaria.

Durante el año de evaluación no se aplicó abonamiento, esporádicamente se aplicaron productos químicos para el control de plagas y enfermedades. En el área de la plantación se cultivaron anteriormente yuca Manihot esculenta y plátano Musa paradisiaca.

Dentro del área de plantación evaluada se registraron un total de 22 especies diferentes al cacao, entre frutales y forestales, tales como bolaina blanca Guazuma crinita (8), bolaina negra Guazuma ulmifolia (3), aguaje Mauritia flexuosa (1), pijuayo Bactris gasipaes (1), cedro colorado Cedrela odorata (2), palta Persea americana (1), plátano Musa paradisiaca (3), guaba Inga edulis (2), capirona Calycophyllum spruceanum (1).
Agroecosistema 2 (denominado "Asociación Cacao-Guaba, AGR 2")

Plantación de seis años de edad de bajo distanciamiento, de $3 \times 3 \mathrm{~m}$, ubicada en condiciones de topografía plana, en la que se realiza deshierbo mecánico cada tres meses, poda de formación, mantenimiento y rehabilitación, abonamiento con roca fosfórica y guano de isla. Se realizan aplicaciones esporádicas del líquido obtenido de la fermentación de cacao para el control de malezas, plagas y enfermedades. El cultivo antecesor al cacao fue el kudzu Pueraria phaseoloides.

Dentro del área de plantación evaluada se registraron un total de 138 especies diferentes al cacao, entre frutales y forestales, tales como guaba Inga edulis (35), lupuna Ceiba sp (1), bolaina negra Guazuma ulmifolia (1), aguaje Mauritia flexuosa (12), tahuarí amarillo Tabebuia serratifolia (2), shebon Attalea butyracea (2), uvilla Pourouma cecropiifolia (1), plátano Musa paradisiaca (40), guayaba Psidium guajava (1), capirona Calycophyllum spruceanum (11), pashaco Schizolobium amazonicum (1).

Tabla 1. Comportamiento de las principales variables meteorológicas temperatura y precipitación durante el periodo experimental.

\begin{tabular}{|c|c|c|c|c|}
\hline \multirow{2}{*}{ Mes } & \multirow{2}{*}{$\begin{array}{l}\text { Precipitaciones } \\
\text { (mm) }\end{array}$} & \multicolumn{3}{|c|}{ Temperaturas $\left({ }^{\circ} \mathrm{C}\right)$} \\
\hline & & Mínima & Máxima & Media \\
\hline Julio & 29,30 & 18,9 & 30,7 & 24,8 \\
\hline Agosto & 125,90 & 18,6 & 31,8 & 25,2 \\
\hline Setiembre & 78,50 & 20,2 & 33,6 & 26,9 \\
\hline Octubre & 133,30 & 21,8 & 32,0 & 26,9 \\
\hline
\end{tabular}

Fuente: Datos de la Estación Meteorológica de Macuya. Universidad Nacional de Ucayali.

Tabla 2. Caracterización de suelo en las parcelas muestreadas (prof. 0-30cm)

\begin{tabular}{lcccl}
\hline \multicolumn{1}{c}{ Agroecosistema } & $\mathbf{p H}$ & $\begin{array}{c}\mathbf{M} . \mathbf{0} \\
(\%)\end{array}$ & $\mathbf{P}(\mathbf{p p m})$ & Textura \\
\hline${ }^{* *}$ Monocultivo & 3,96 & 2,91 & 5,77 & Franco arcilloso \\
${ }^{*}$ Asociación Cacao - Guaba & 4,54 & 1,69 & 1,10 & Franco Arenoso \\
${ }^{*}$ Agroforestal con cobertura de kudzu & 4,60 & 2,60 & 1,90 & Franco \\
\hline
\end{tabular}

* Laboratorio de suelos de análisis de suelos, plantas agua y fertilizantes de la Universidad Nacional Agraria la Molina.

** Laboratorio de análisis de suelos de la Universidad Nacional Agraria de la Selva. 
Agroecosistema 3 (denominado "Agroforestal con cobertura, AGR-3")

Plantación de siete años de edad bajo distanciamiento de $3 \times 3 \mathrm{~m}$, establecido con cobertura de kudzu Pueraria phaseloides, ubicada en condiciones de topografía ligeramente ondulada; en la que se realiza deshierbo mecánico cada dos meses, poda de mantenimiento, abonamientos esporádicos de guano de isla y roca fosfórica, no se aplicaron productos químicos para el control de plagas y enfermedades. Los cultivos antecesores al cultivo de cacao fueron los cultivos de arroz Oryza sativa, maíz Zea maízy y látano Musa paradisiaca.

Dentro del área de plantación evaluada se registraron un total de 150 especies diferentes al cacao, entre frutales y forestales, tales como guaba Inga edulis (38), bolaina blanca Guazuma crinita (3), yarina Phytelephas macrocarpa (12), aguaje Mauritia flexuosa (1), pijuayo Bactris gasipaes (1), cedro Cedrela odorata (1), palta Persea americana (1), plátano Musa paradisiaca (23), shebon (15), capirona Calycophyllum spruceanum (2), sangre de grado Croton lechleri (9), caimito Pouteria caimito (1), caoba Swietenia macrophylla (9).

Material vegetal. Las plantas de cacao evaluadas en cada plantación corresponden a plantas provenientes de patrones criollos e injertados con el clon trinitario CCNN 51.

Muestreo de suelo. Se empleó un área efectiva de $7500 \mathrm{~m}^{2}$ por agroecosistema de cacao, en los que se realizó muestreo sistemático; se seleccionaron 15 plantas de cacao al azar en zigzag y se tomaron tres muestras de suelo equidistantes, a la altura de la proyección de la copa a una profundidad de $0-20 \mathrm{~cm}$, de las cuales se obtuvo una muestra compuesta por planta. El muestreo se realizó con una periodicidad de 30 días.

Cuantificación de esporas. Se tomó una muestra de $10 \mathrm{~g}$ de cada una de las 45 muestras de suelo obtenidas por evaluación, las cuales fueron procesadas en el Laboratorio de Entomología, Pruebas Biológicas y Bioterio del Instituto de Investigaciones de la Amazonía Peruana, para el aislamiento y conteo de esporas de los de HMA, según el método descrito por Ruiz y Rojas (2011), basado en los métodos de Gerdemann y Nicolson (1963) y Sieverding (1983), que consistió en el tamizado y decantado por vía húmeda de los propágulos del hongo, los que se recolectaron sobre una malla de $45 \mu \mathrm{m}$ de apertura y se separaron por centrifugación (Centrífuga: marca Hettich, modelo
Rotina $420 \mathrm{R}$ ) con una gradiente de sacarosa al $45 \%$. El conteo de esporas se realizó usando un estereoscopio a 50x de aumento (Estereoscopio: Trinocular marca Carlz-Zeiss, modelo Stemi 2006).

Identificacion de especies HMA. De cada sistema de cultivo, se envió a Alemania, para su análisis, una muestra representativa y compuesta de suelo de aproximadamente $05 \mathrm{~kg}$. El análisis fue realizado por el Dr. E. Sieverding. Las muestras se homogenizaron y se separaron las esporas presentes en $50 \mathrm{~g}$ de suelo de cada ecosistema. Las esporas se aislaron bajo el esteroscopio y se montaron en placas de vidrio de microscopía. Las esporas se fijaron permanentemente en una solución de PVLG (glicoglicerina de alcohol polivenilico). Con $400 \mathrm{x}$ aumento en el microscopio de trasmision de luz se observaron las esporas y se clasificaron morfologicamente a nivel de género y especie, siguiendo la clasificación propuesta por Oehl et al (2011).

Procedimiento estadístico. Para el análisis de los resultados obtenidos se empleo el paquete estadístico IBM SPSS STATISTICS, analizando los promedios mensuales, haciendo uso de la prueba de Tukey $(p<0,05)$, para lo cual se emplearon previamente las pruebas de Normalidad (Shapiro Wilk) y Levene. Para la generación de gráficos se empleó el diagrama de cajas.

\section{RESULTADOS}

\section{Abundancia de esporas de hongos de micorriza arbuscular}

Los resultados del promedio mensual del número de esporas por parcela se indican en la tabla 4. El análisis de comparación de medias registró diferencias significativas en la abundancia de HMA para el AGR-3, comparado con los AGR-1 y AGR-2, con media superior de 917,5. El análisis de variación del promedio mensual de 15 plantas evaluadas para cada agroecosistema se presenta en el diagrama de caja del Gráfico 1, en el que se observa mayor valor promedio para el AGR-3.

\section{Diversidad de hongos de micorriza arbuscular}

Los resultados de la identificación de HMA presentes en cada agroecosistema se muestran en la tabla 5. Se observa mayor diversidad de especies en el AGR-2, seguido del AGR-1 y AGR-3, respectivamente. 
Tabla 3. Significancia estadística del factor evaluado sobre el número de esporas.

\begin{tabular}{|c|c|c|c|}
\hline Prueba & Agroecosistema & Agroecosistema & Sig. \\
\hline \multirow{6}{*}{ HSD de Tukey } & \multirow{2}{*}{$A G R-1$} & AGR - 2 & ,999 \\
\hline & & AGR - 3 &, 003 \\
\hline & \multirow{2}{*}{$A G R-2$} & AGR - 1 & 999 \\
\hline & & AGR - 3 & ,002 \\
\hline & \multirow{2}{*}{$A G R-3$} & AGR - 1 &, 003 \\
\hline & & AGR - 2 &, 002 \\
\hline
\end{tabular}

Tabla 4. Efecto del agroecosistema en el número de esporas/100 gr de suelo (valores medios \pm error típico)

\begin{tabular}{cc}
\hline $\begin{array}{c}\text { Factor } \\
\text { Agroecosistema }\end{array}$ & Número de esporas \\
\hline AGR -1 & $542,5( \pm 37,05)^{\mathrm{a}}$ \\
AGR -2 & $540,0( \pm 51,64)^{\mathrm{a}}$ \\
AGR -3 & $917,5( \pm 71,69)^{\mathrm{b}}$ \\
\hline
\end{tabular}

* Los valores con diferente letra, para efectos principales del factor, difieren significativamente $(p<0,05)$.

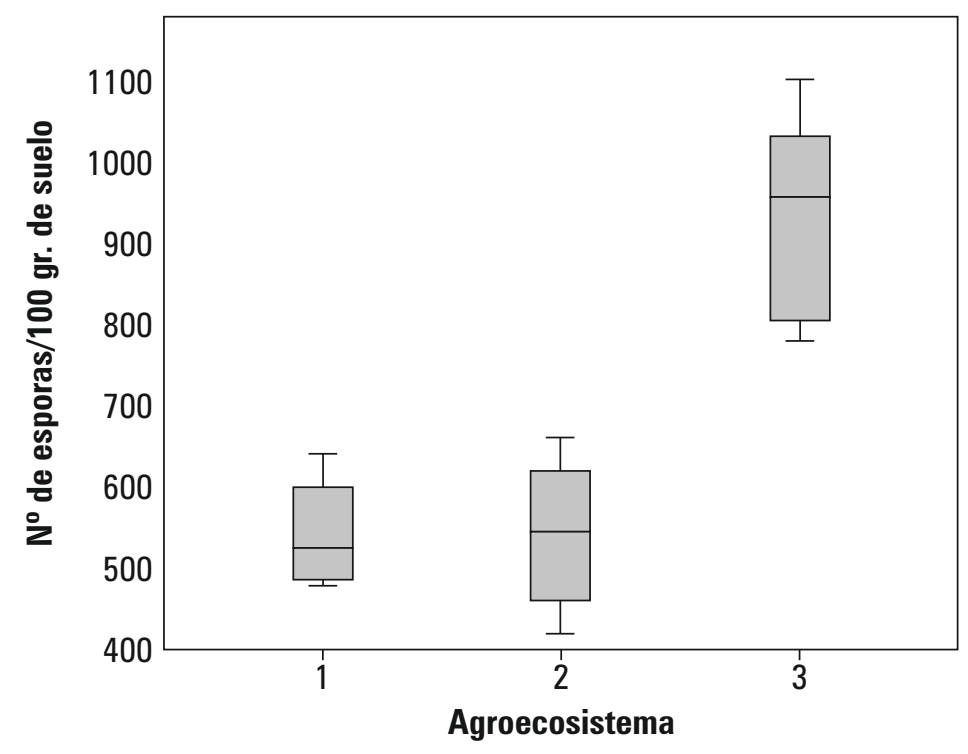

Gráfico 1. Diagrama de caja del promedio mensual de cuatro evaluaciones por agroecosistema. 
Tabla 5. Especies de hongos de micorriza arbuscular en tres agroecosistemas de cacao.

\begin{tabular}{|c|c|c|}
\hline \multicolumn{3}{|c|}{ Agroecosistemas } \\
\hline 1 & 2 & 3 \\
\hline Acaulospora puntata & Acaulospora longua & Acaulospora scrobiculata \\
\hline Acaulospora spinossisima & Acaulospora mellea & Acaulospora sp. $2^{*}$ \\
\hline Acaulospora spinosa & Acaulospora rehmii & Acaulospora sp. $3^{*}$ \\
\hline Acaulospora sp. $1^{*}$ & Acaulospora scrobiculata & Ambispora gerdemannii \\
\hline Ambispora brasiliensis & Acaulospora tuberculata & Diversispora spurca \\
\hline Archeospora trappei & Acaulospora sp. $4^{*}$ & Diversispora lutea \\
\hline Claroideoglomus etunicatum & Acaulospora sp. $5^{*}$ & Fuscutata heterógama \\
\hline Diversispora spurca & Acaulospora sp. $6^{*}$ & Glomus diaphanum \\
\hline Glomus fasciculatum & Archeospora trappei & Glomus sp. $1^{*}$ \\
\hline Glomus sp. $1^{*}$ & Archeospora undulate & Glomus sp. $2^{*}$ \\
\hline Glomus sp. $2^{*}$ & Cetraspora pellucida & Pacispora dominikii \\
\hline Glomus sp. $3^{*}$ & Claroideoglomus claroideum & Sclerocystis rubiforme \\
\hline Pacispora sp. $1^{*}$ & Diversispora spurca & ${ }^{*}$ Morfotipo 1 sin identificar \\
\hline Paraglomus brasiliensis & Diversispora versiforme & \\
\hline Sclerocystis coremioides & Diversispora sp. $1^{*}$ & \\
\hline \multirow[t]{14}{*}{ *Morfotipo 1 sin identificar } & Fuscutata heterógama & \\
\hline & Glomus brohultii & \\
\hline & Glomus fasciculatum & \\
\hline & Glomus sp. $1^{*}$ & \\
\hline & Glomus sp. $4^{*}$ & \\
\hline & Glomus sp. $5^{*}$ & \\
\hline & Kuklospora kentinenis & \\
\hline & Kuklospora sp. $1^{*}$ & \\
\hline & Kuklospora sp. $2^{*}$ & \\
\hline & Pacispora sp. $2^{*}$ & \\
\hline & Paraglomus occultum & \\
\hline & Paraglomus sp. & \\
\hline & Sclerocystis rubiforme & \\
\hline & Sclerocystis sp. $1^{*}$ & \\
\hline
\end{tabular}

Identificado por Ewald Sieverding, 2013.

* Especies no identificadas, probablemente nuevas para la ciencia.

* De acuerdo con la última clasificación taxonómica de los HMA (Oehl et al., 2011). 


\section{DISCUSIÓN}

En AGR-1 se obtuvo un rango del número de esporas (Gráfico 1), que varió de 480 a 640 esporas por 100 gr de suelo; en el AGR-2 el número de esporas varió de 420 a 660 esporas por 100 gr de suelo y en el AGR-3 el número de esporas varió de 780 a 1100 esporas por 100 gr de suelo, factor asociado a la cobertura con Kudzu. Se aprecian diferencias significativas en la abundancia de HMA, por tanto, se afirma que existen diferencias entre la abundancia de esporas según el tipo de agroecosistema. Los resultados indican una media superior en el AGR-3, en el estudio realizado por Crews and Peoples (como se cita en Del Carmen Pérez, et al. 2012), se afirma que el efecto positivo del abono verde como cultivo de cobertura sobre la riqueza de especies de HMA y el índice de diversidad, se debe principalmente a que estos mejoran la actividad biológica del suelo. En el estudio realizado por Espíndola et al. y Rivera et al. (como se cita en Martín, Rivera, Arias y Rentería. 2009), mencionan que algunas prácticas como el abonado verde pueden aumentar la presencia de los propágulos nativos de HMA en el suelo. El uso de leguminosas como cobertura, incrementa la población nativa de HMA de dos a tres veces. Deguchi (como se cita en Del Carmen Pérez, et al. 2012) hace referencia a la influencia en la riqueza de especies y en su diversidad (Del Carmen Pérez, et al. 2012). Blanco y Gutiérrez (1998) mencionan que Mucuna sp., favoreció el incremento de las poblaciones de esporas del género Glomus principalmente. Asimismo, Sancho y Cervantes (1997) reportan que Mucuna sp. , al ser una planta altamente competitiva, modifica la composición florística del agroecosistema, influyendo en la composición de especies de HMA (Blanco y Gutiérrez 1998). También, Del Carmen Pérez, et al. (2012), concluyen que la presencia de cobertura de Mucuna $s p$ afectó positivamente a la riqueza y diversidad de HMA observadas en parcelas de maíz, encontrando 122,7 esporas en 50 gr de suelo.

En cuanto a las especies de HMA identificados por agroecosistema (Tabla 5), se reporta de las 46 especies, encontrando solo dos especies en común para los tres agroecosistemas, Diversispora spurca y un morfotipo del género Glomus; evidenciando diferencias entre las comunidades encontradas. Así mismo, se reportan 6 especies en común con las encontradas por Ruiz, Rojas y Sieverding, 2011, en muestras de suelo procedentes de parcelas de las zonas de Aguaytía y Nueva Requena. Todo esto reafirma la existencia de un gran potencial diverso de especies de HMA en suelos amazónicos. Además, Ruiz, Rojas y Sieverding, 2011, mencionan que estas diferencias pueden estar relacionadas con las características químicas de los suelos, particularmente la acidez y el contenido de nutrientes. Asimismo, la precipitación pluvial, además de la composición florística en cada localidad, pudo haber influido también en la ocurrencia de las especies de HMA.

\section{CONCLUSIONES}

Se encontró mayor abundancia de esporas en la parcela de cacao bajo el sistema con cobertura, con un valor mínimo de 780 esporas y un valor máximo de 1100 esporas por 100 gr. de suelo. Los datos obtenidos indican una mayor abundancia de la población de HMA en presencia de Pueraria phaseoloides. Esto constituye un potencial para el incremento de propágalos de HMA dentro del sistema, lo cual contribuiría en la mejora de la eficiencia de los nutrientes en suelos ácidos, siempre que se propicie la integración de ambas técnicas. Se reportaron 46 especies de HMA en la zona de estudio, con dos especies en común para cada agroecosistema: Diversispora spurca y un morfotipo del género Glomus.

\section{AGRADECIMIENTOS}

Los autores expresan su profundo agradecimiento al Dr. Pedro Ruiz Cubillas, en nuestra memoria perduran su apoyo incondicional y dedicación al trabajo, además de habernos honrado hasta el final de sus días con su acompañamiento profesional y cercanía personal. Se agradece a la Asociación de Cacaoteros Tecnificados de Padre Abad (ACATPA), por las facilidades brindadas para el desarrollo del estudio en parcelas pertenecientes a los socios: Anahuario Fasabi Tapullima, Miguel Navarro Mass y Almeyda David Mori Flores, a quienes expresamos nuestro agradecimiento por todo el apoyo brindado, a los técnicos Carlos Marín Pérez, Cesar Young Salva y al Bachiller Adrián Cabana Cayllahua por el apoyo brindado en la extracción de muestras durante el trabajo de campo. A la Universidad Nacional de Ucayali por el apoyo brindado para contar con los datos meteorológicas de la zona en estudio.

\section{REFERENCIAS BIBLIOGRÁFICAS}

Barrer, S. 2009. El uso de hongos micorrizicos arbusculares como una alternativa para la agricultura. Revista Biotecnología en el Sector Agropecuario y Agroindustrial, 7 (1): 123-132.

Blanco, F. \& R. Gutiérrez. 1998. Efecto de la Mucuna sp. en la composición de la comunidad 
de hongos MA del suelo y en la respuesta del maíz a la inoculación con hongos MA. Agronomía Costarricense, 22 (2): 153-161.

Blanco, F. \& E. Salas. 1997. Micorrizas en la Agricultura: Contexto mundial e investigación realizada en Costa Rica. Agronomía Costarricense, 21 (1): 55-67.

Del Carmen Pérez L. Y, Álvarez S. J., Mendoza V. J, Pat-F. J. M, Gómez A. R. \& Cuevas L. 2012. Diversidad de hongos micorrícicos arbusculares en maíz con cultivo de cobertura y biofertilizantes en Chiapas, México. Gayana Botánica, 69 (1): 46-56.

Flores B. Y. 2010. Crecimiento y productividad de plantaciones forestales en la amazonía peruana. Instituto Nacional de Innovación Agraria INIA.

Iglesias, L., Salas, E., Leblanc, H.A. \& Nygren. P. 2010. Morfología de los hongos micorrícicos arbusculares en las raíces de Theobroma cacao e Inga edulis en un experimento de inoculaciónz cruzada. Tierra Tropical, 6(1):33-44.

Martín G. M, Rivera R., Arias L. \& Rentería M. 2009. Efecto de la Canavalia ensiformis y micorrizas arbusculares en el cultivo del maíz. Revista Cubana de Ciencia Agrícola, 43 (2): 191-199.

Medina, A. \& R. Azcón. 2010. Effectiveness of the application of arbuscular mycorrhizal fungi and organic amendments to improve soil quality and plant performance under stress conditions. Journal of soil science and plant nutrition, 10 (3): 354-372.

Oehl, F., Alves da Silva G., Tomio Goto, B. \& Sieverding, E. 2011.Glomeromycota: three new genera and glomoid species reorganized. Mycotaxon, 116: 75-120. doi: 10.5248/116.75.

Ruiz, P.O. \& Rojas, K.C. 2011. Hongos de micorriza arbuscular. Protocolos y procedimientos de evaluación. Manual técnico. Pucallpa. Perú.

Ruiz, P.O., Rojas, K.C. \& Sieverding, E. 2011. La distribución geográfica de los hongos de micorriza arbuscular: una prioridad de investigación en la Amazonía peruana. Espacio y Desarrollo, 23: 47-63.

Sancho, F. \& C. Cervantes. 1997. El uso de plantas de cobertura en sistemas de producción de cultivos perennes y anuales en Costa Rica. En: F. Bertsch, W. Badilla \& E. Bornemisza (eds.), Congreso Nacional Agronómico y de Recursos Naturales, Vol. 3, Suelos, p. 181-188. UNED y EUNA. San José, Costa Rica.

Recibido: 19/05/2014

Aceptado para publicación: 18/07/2014 